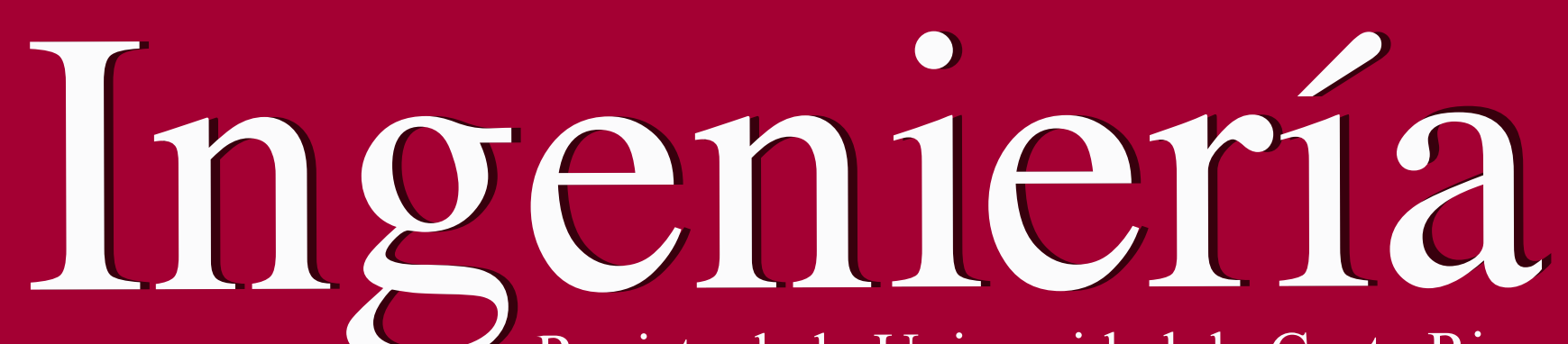

Revista de la Universidad de Costa Rica JULIO / DICIEMBRE 2020 - VOLUMEN 30 (2)

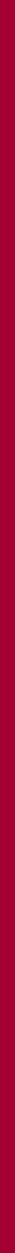




\title{
Análisis en Modo de Conducción Discontinuo del convertidor DC/DC 1-FB ${ }^{-1}$
}

\author{
1-FB-1 DC-DC Converter Analysis in Discontinuous Conduction Mode \\ Luis Alejandro Gólcher Barguil \\ Docente de la Escuela de Ingeniería Eléctrica \\ Universidad de Costa Rica, San José, Costa Rica \\ luis.golcher@ucr.ac.cr \\ ORCID: https://orcid.org/0000-0002-6260-0525
}

Recibido: 6 de enero 2020

Aceptado: 24 de abril 2020

\section{Resumen}

Se plantea el análisis del nuevo Convertidor DC-DC denominado 1-FB-1 el cual opera en modo de conducción discontinuo. Se establece el límite de operación entre modos de conducción continuo y discontinuo para definir apropiadamente los valores de los elementos en la etapa de diseño. El análisis abarca tanto la descripción de los estados del circuito, así como expresiones analíticas que describen la corriente del inductor y el voltaje de salida del capacitor.

Palabras clave:

Convertidor PWM, Fuente de poder DC, Enfoque minima corriente, Transformación DC

\begin{abstract}
A Discontinuous Conduction Mode analysis of the new 1-FB ${ }^{-1} \mathrm{DC} / \mathrm{DC}$ converter is proposed. The article establishes the limits for this mode to appropriately define the continuous or discontinuous operation during the designing stage. This analysis encompasses the analytical expressions of the converter inductor current and the output voltage waveforms
\end{abstract}

Keywords:

PWM converter, DC Power Supply, Minimum Current Approach, DC transformation 


\section{INTRODUCCIÓN}

Los convertidores DC-DC con conmutación PWM pueden ser operados en modo de conducción continuo o discontinuo, o bien en el límite. En el modo de conducción discontinuo, la energía almacenada en el inductor es transferida completamente durante cada ciclo de conmutación. Nandankar y Rothe (2016) indican que el modo de conducción discontinuo permite alcanzar una alta densidad de potencia al minimizar el tamaño del inductor. De esta forma, se logra disminuir el tamaño físico de la fuente de poder.

El modo de conducción discontinuo ocurre cuando la corriente del inductor intenta cambiar de sentido, pero la implementación del switch no se lo permite y permanece en cero el resto del periodo de conmutación. Motto (1977) y Mohan, Undeland y Robbins (2003) apuntan que el estrés eléctrico sobre el transistor se reduce al encenderse con cero corriente. Adicionalmente, Tse, Shu-hung, Hui y So (2000) señalan que el modo de conducción discontinuo permite mejorar la estabilidad del convertidor y así brindar una operación más estable en lazo cerrado. Lin y Chen (1994) resaltan que en modo de conducción discontinuo se pueden aplicar técnicas de variación aleatoria de frecuencias de conmutación para mitigar la interferencia electromagnética.

Erickson y Maksimovic (2001) mencionan que las propiedades del convertidor cambian considerablemente en el modo de conducción discontinuo. La razón de conversión DC depende ahora de la carga y los parámetros del circuito, lo que no ocurre en la operación continua. Krein (1998) y Rashid (1995) indican que el modo de conducción discontinuo se puede mantener ante variaciones de la carga, regulando la frecuencia de conmutación para sumar una disminución en EMI.

El propósito de este artículo es establecer un marco de análisis del modo de conducción discontinuo en el convertidor DC-DC 1-FB-1 para mejorar la eficiencia de los convertidores con aislamiento galvánico. La topología del convertidor DC-DC 1-FB-1 fue propuesta por Gólcher (2014), así como su análisis en modo de conducción continuo. El circuito se muestra en la Figura 1.

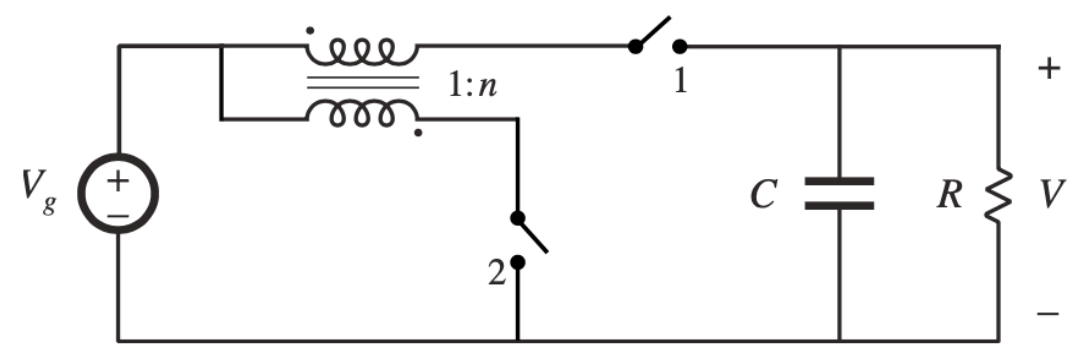

Figura 1. Topología del Convertidor 1-FB-1. Gólcher (2014). 


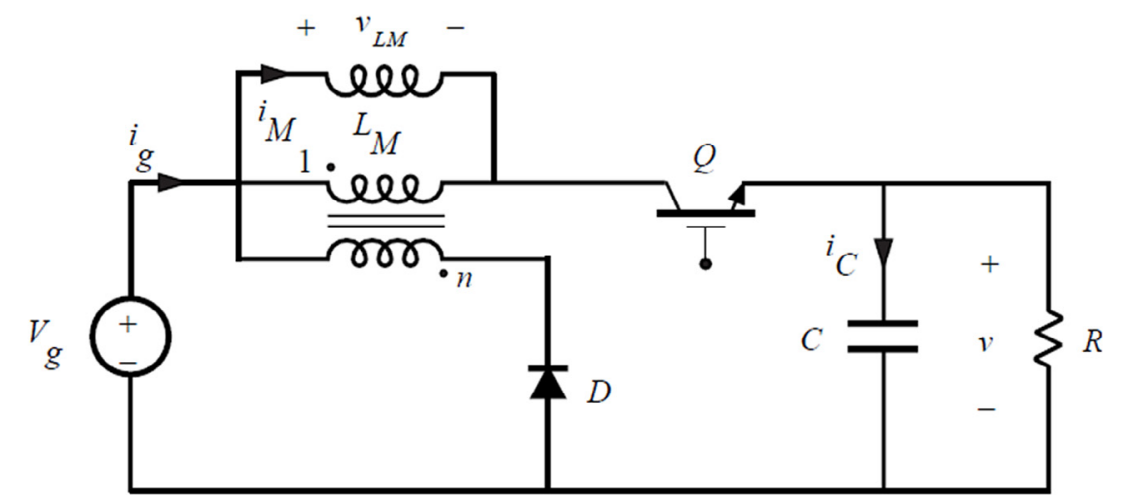

Figura 2. Topología del Convertidor 1-FB-1 con interruptores reales. Fuente: Gólcher (2014).

Gólcher (2014) propuso una implementación práctica con los interruptores que se indican en la Figura 2, en donde $\mathrm{L}_{\mathrm{M}}$ representa la inductancia magnetizante.

La importancia de este trabajo es presentar un Convertidor DC-DC PWM con características para bajar el voltaje de entrada, minimizar las pérdidas en los semiconductores al operador en modo de conducción discontinuo y disminuir el tamaño del circuito al obtener un menor inductor con cargas altas.

\section{ANÁLISIS DEL RANGO VÁLIDO DEL CICLO DE TRABAJO}

Gólcher (2014) determina que en modo de conducción continuo el convertidor se rige por las siguientes ecuaciones.

El voltaje de salida, $V$, está dado por:

$$
V=\left(1-\frac{D^{\prime}}{n D}\right) V_{g}
$$

La corriente de la inductancia magnetizante, $I_{M}$, es:

$$
I_{M}=\frac{1}{D}\left(1-\frac{D^{\prime}}{n D}\right) \frac{V_{g}}{R}
$$

El rizado de la corriente de la inductancia magnetizante, $\Delta i_{M}$, es igual a:

$$
\Delta i_{M}=\frac{D^{\prime} V_{g}}{2 L_{M} f_{s}}
$$


La forma de onda de la corriente de la inductancia magnetizante se muestra en la Figura 3.

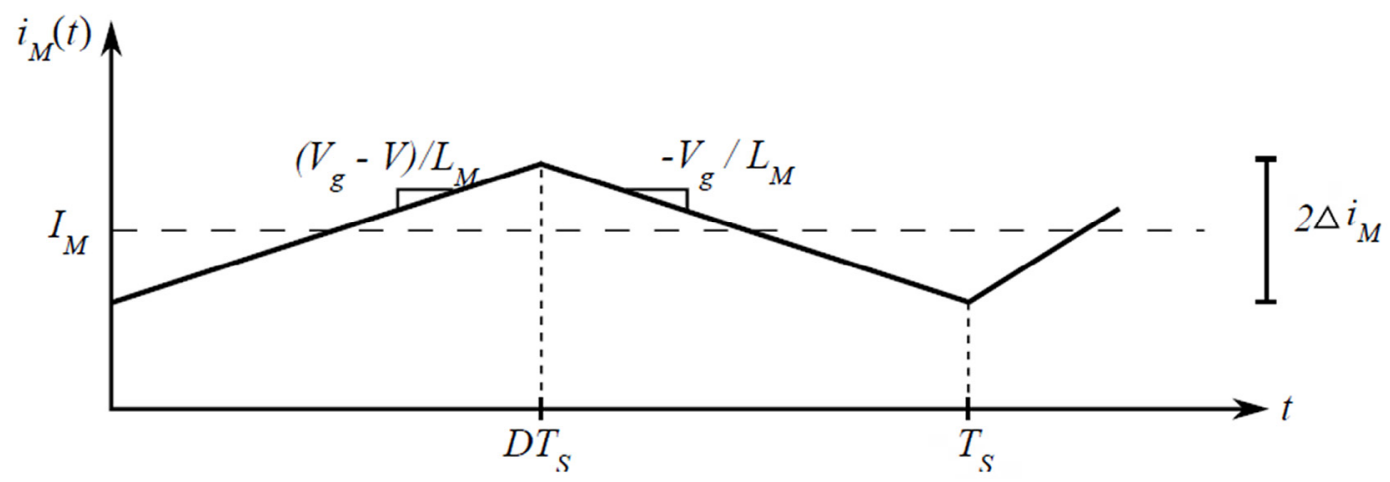

Figura 3. Forma de onda de $i_{M}$. Fuente: el autor.

Para que el diodo se encienda durante el segundo intervalo del periodo de conmutación, una vez que el trasistor se ha encendido por $0<t \leq D T_{s}$, se requiere que la corriente de la inductancia magnetizante sea positiva, $I_{M}>0$, o sea que:

$$
\underset{>0}{\frac{1}{D}}\left(1-\frac{D^{\prime}}{n D}\right) \underset{>0}{\frac{V_{g}}{R}}>0
$$

Para que se cumpla la desigualdad de la ecuación (4), la expresión entre paréntesis debe ser positiva, así:

$$
1-\frac{D^{\prime}}{n D}>0
$$

Si $n=1$, se tiene que, $D^{\prime}<D$, y como $D^{\prime}=1-D$, entonces

$$
D>1 / 2
$$

Para $n>1$, de la ecuación (5) se tiene que:

$$
\begin{aligned}
& D n>1-D \\
& \Rightarrow D n+D>1
\end{aligned}
$$

despejando $D$ :

$$
D>\frac{1}{1+n}
$$


El resultado de la ecuación (9) es consistente con el caso de $n=1$. La Tabla 1 muestra el rango válido de $D$ y la tensión DC de salida para varios valores de $n$.

Tabla 1. Expresiones de la tensión de salida DC para varios valores de $n$

\begin{tabular}{lcc}
\hline$n$ & Rango $D$ & Tensión $V$ \\
\hline 1 & $D>\frac{1}{2}$ & $V=\left(1-\frac{D^{\prime}}{D}\right) V_{g}$ \\
2 & $D>\frac{1}{3}$ & $V=\left(1-\frac{D^{\prime}}{2 D}\right) V_{g}$ \\
3 & $D>\frac{1}{4}$ & $V=\left(1-\frac{D^{\prime}}{3 D}\right) V_{g}$ \\
$\frac{1}{2}$ & $D>\frac{2}{3}$ & $V=\left(1-\frac{2 D^{\prime}}{D}\right) V_{g}$ \\
$\frac{1}{3}$ & $D>\frac{3}{4}$ & $V=\left(1-\frac{3 D^{\prime}}{D}\right) V_{g}$ \\
\hline
\end{tabular}

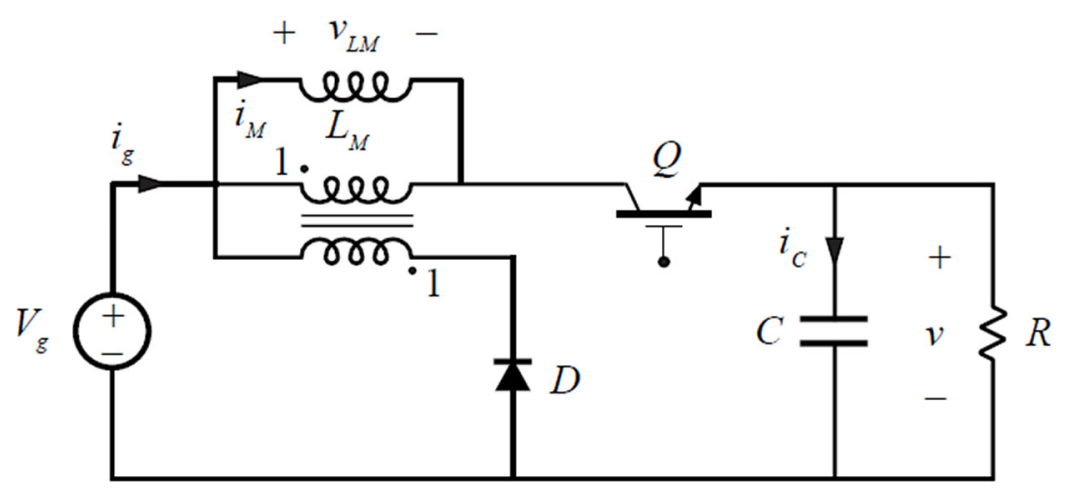

Figura 4. Convertidor $\mathrm{FB}^{-1}$ con $n=1$. Fuente: Elaboración propia.

El análisis se continúa para el caso de $n=1$, ya que para otros valores los análisis son similares. La topología del convertidor se muestra en la Figura 4. Se asume que los elementos están libres de pérdidas y que se está operando en estado estacionario. 


\section{LÍMITE ENTRE MODOS DE CONDUCCIÓN}

El límite entre los modos de conducción continuo y discontinuo se determina analizando la desigualdad establecida en (10), ya que cuando la corriente DC, $I_{M}$, es menor a su rizado, $\Delta i_{M}$, la corriente intentará ser negativa y provocará que el diodo se apague prematuramente. Esta forma de expresar el límite de los modos de conducción se conoce como el método de $\mathrm{K}_{\text {y }} \mathrm{K}_{\text {crit }}$, analizado en Erickson y Maksimovic (2001). Si la inecuación (10) es verdadera, entonces se opera en Modo de Conducción Discontinuo.

$$
I_{M}<\Delta i_{M}
$$

Primero se sustituye (2) y (3) en la desigualdad (10):

$$
\frac{1}{D}\left(1-\frac{D^{\prime}}{D}\right) \frac{V_{g}}{R}<\frac{D^{\prime} V_{g}}{2 L_{M} f_{s}}
$$

Re-escribiendo:

$$
\underbrace{\frac{2 L_{M}}{R T_{S}}}_{K}<\frac{D^{\prime} D^{2}}{D-(1-D)}=\underbrace{\frac{D^{\prime} D^{2}}{2 D-1}}_{K_{\text {crit }}}
$$

El valor de $K$ solamente depende de parámetros del circuito, mientras que $\mathrm{K}_{c r i t}$ depende únicamente del ciclo de trabajo, $D$. En la Figura 5 se grafica $\mathrm{K}_{\text {crit }}$ para el rango válido de $D$.

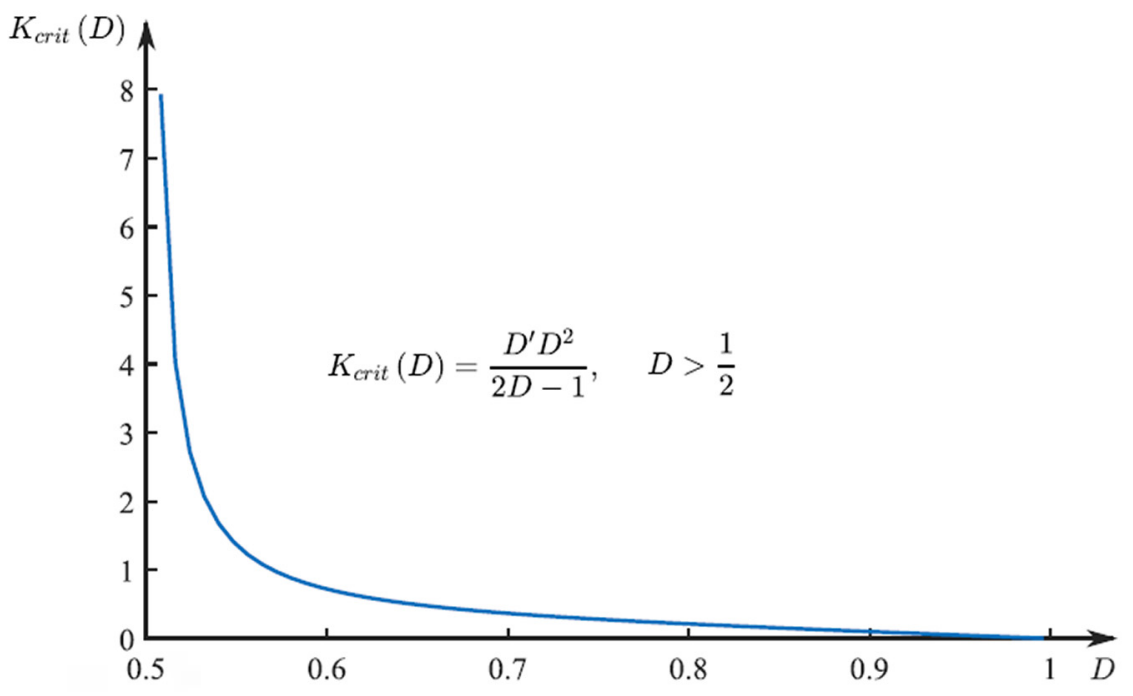

Figura 5. Gráfica de $\mathrm{K}_{\text {crit }}$ para $D>1 / 2$. Fuente: Elaboración propia. 
Como ejemplo, si $K$ fuese igual a 2, entonces gráficamente se puede observar el límite entre modos de conducción conforme el ciclo de trabajo va de $1 / 2$ a 1 , como se ve en la Figura 6.

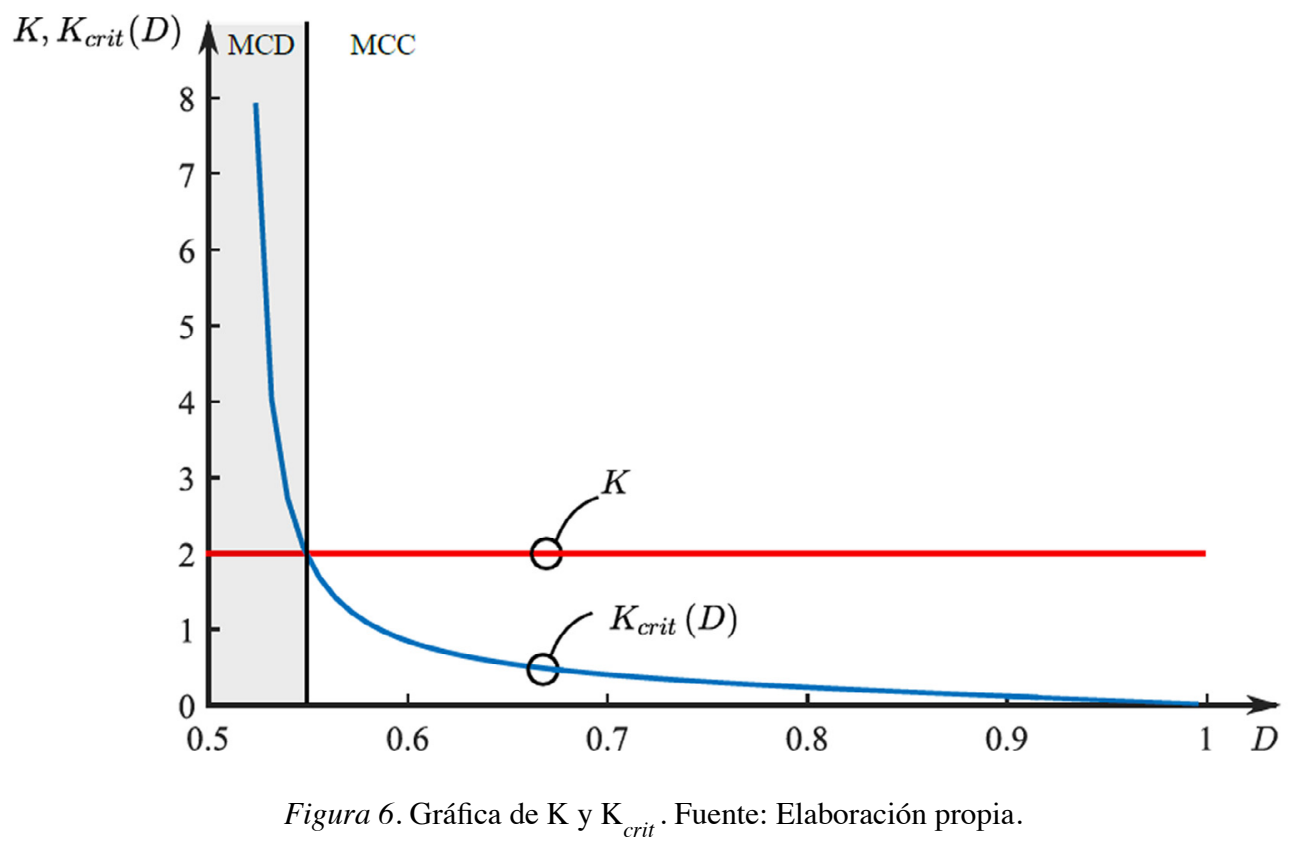

Si D opera cerca de 1/2, la probabilidad de estar en Modo de Conducción Discontinuo es mayor.

\section{ANÁLISIS EN MODO DE CONDUCCIÓN DISCONTINUO}

El análisis en Modo de Conducción Discontinuo (MCD) se realiza para el caso $n=1$ en la Figura 4. Para realizar el análisis, se utiliza la metodología establecida por Middlebrook y Çuk (1983). En el estado estacionario del MCD se presentan tres intervalos por cada ciclo de conmutación. En el primer intervalo, el transistor se enciende durante el ciclo de trabajo, $D_{1}$. Posteriormente, el transistor se apaga por el circuito de control; la corriente de la inductancia magnetizante comienza a disminuir al encenderse el diodo. El segundo intervalo termina cuando la corriente de la inductancia magnetizante intenta pasar a negativo, pero el diodo no la deja al apagarse. El tercer intervalo inicia con todos los semiconductores apagados, tanto el diodo como el transistor. Éste último intervalo finaliza cuando el circuito de control inicia un nuevo ciclo de conmutación al encender el transistor $Q$. El análisis de los tres intervalos se detalla a continuación.

Intervalo I: Transistor $Q$ encendido y Diodo $D$ apagado.

En el primer intervalo el circuito de control enciende el transistor y el diodo se despolariza, ya que no puede conducir corriente negativa. En la Figura 7 se muestra la topología resultante. La tensión $v_{L M}$ y la corriente $i_{C}$, se determinan según las ecuaciones: 


$$
\begin{aligned}
& v_{L M} \cong V_{g}-V \\
& i_{C} \cong i_{M}-V / R
\end{aligned}
$$

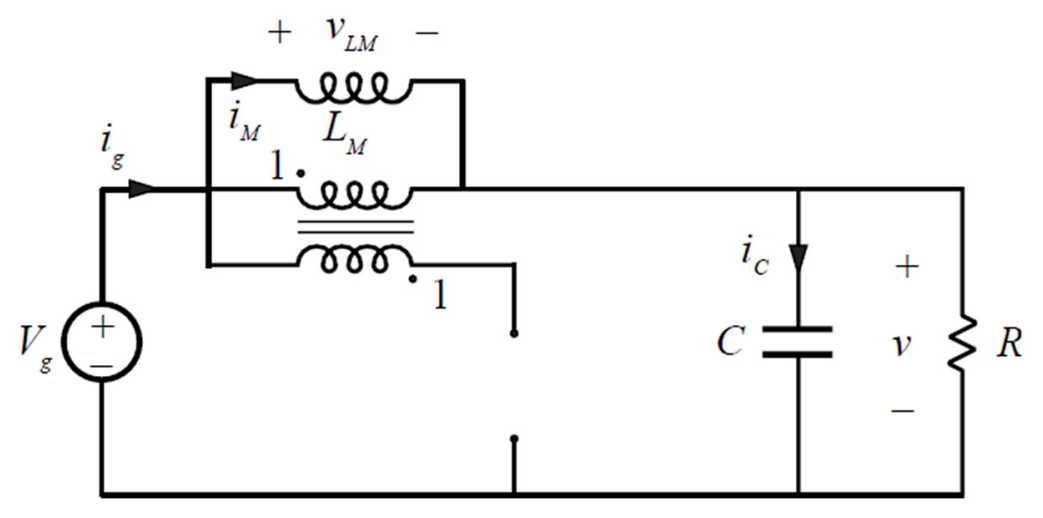

Figura 7. Topología intervalo I con $n=1$. Fuente: Elaboración propia.

Intervalo II: Transistor $Q$ apagado y Diodo $D$ encendido.

La topología resultante se muestra en la Figura 8. El circuito de control apaga el transistor y el diodo se enciende para conducir la corriente $i_{M}$. La tensión $v_{L M}$ y la corriente $i_{C}$, se determinan según las ecuaciones:

$$
\begin{aligned}
& v_{L M}=-V_{g} \\
& i_{C} \cong-V / R
\end{aligned}
$$

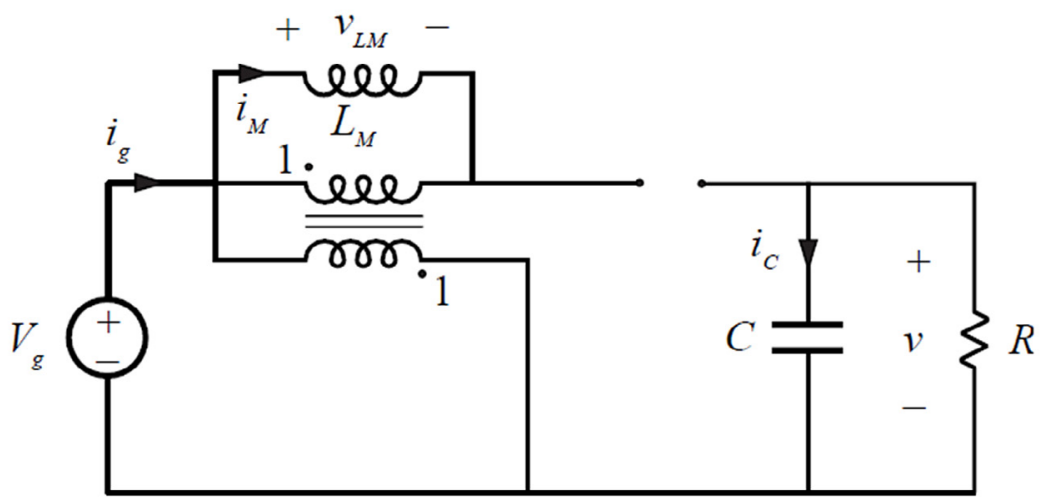

Figura 8. Topología intervalo II con $n=1$. Fuente: Elaboración propia.

Intervalo III: Transistor $Q$ y Diodo $D$ apagados.

En este intervalo tanto el diodo como el transistor se encuentran apagados. La topología para este intervalo de análisis se muestra en la Figura 9. El diodo termina en el segundo intervalo apagado, 
ya que la corriente de la inductancia magnetizante disminuye a cero. De la ley de tensiones y de corrientes de Kirchhoff se tiene que:

$$
\begin{aligned}
& v_{L M}=L_{M} \frac{d 0}{d t}=0 \quad \text { ya que } i_{M}=0 \\
& i_{C} \cong-V / R
\end{aligned}
$$

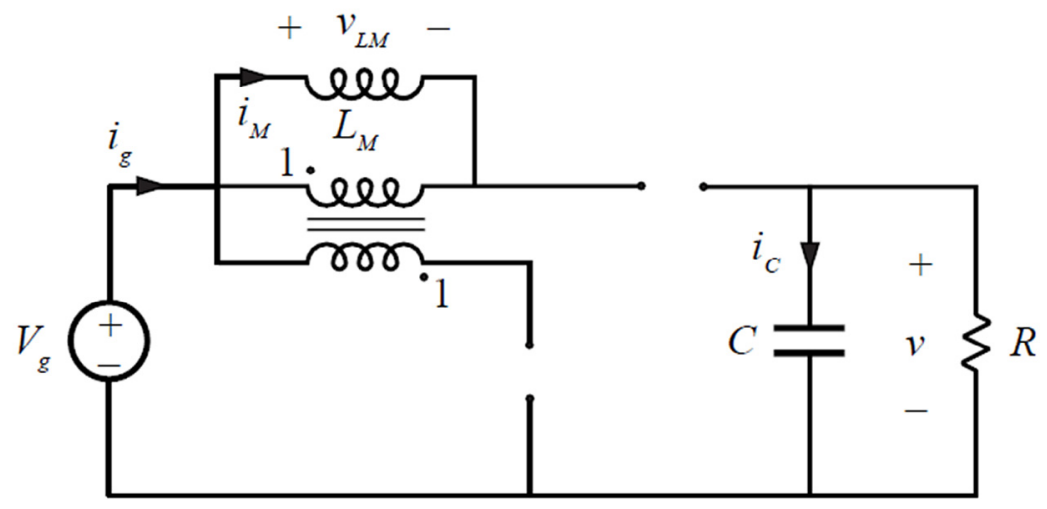

Figura 9. Topología intervalo III. Fuente: Elaboración propia.

Realizando el Balance de Volts/s en $L_{M}$ se obtiene la expresión para $D_{2}$, ecuación (21):

$$
\begin{aligned}
& \left\langle v_{L M}\right\rangle=D_{1}\left(V_{g}-V\right)+D_{2}\left(-V_{g}\right)+D_{3}(0)=0 \\
& D_{2}\left(V_{g}\right)=D_{1}\left(V_{g}-V\right) \\
& D_{2}=\frac{D_{1}}{V_{g}}\left(V_{g}-V\right)
\end{aligned}
$$

Se procede con el Balance de Amp/s en el nodo indicado en la Figura 10:

$$
i_{Q}=i_{C}+v / R
$$

promediando el nodo:

$$
\begin{aligned}
& \left\langle i_{Q}\right\rangle=\underbrace{\left\langle i_{C}\right\rangle}_{=0}+\langle v / R\rangle \\
& \left\langle i_{Q}\right\rangle=\langle v / R\rangle=V / R
\end{aligned}
$$




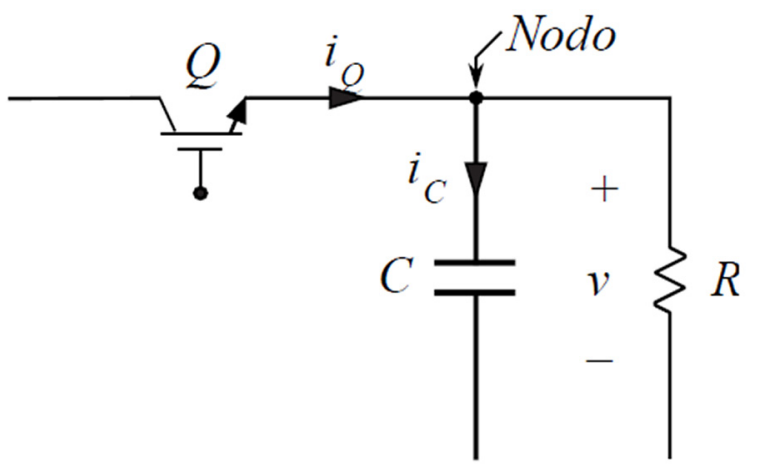

Figura 10. Circuito para determinar $i_{C}$. Fuente: Elaboración propia

Para averiguar $\left\langle i_{Q}\right\rangle$, se grafica $i_{M}$ en la Figura 11. La corriente en el transistor es únicamente igual a la corriente de la inductancia magnetizante durante el primer intervalo; en el resto del periodo de conmutación es cero, ya que el transistor está apagado.

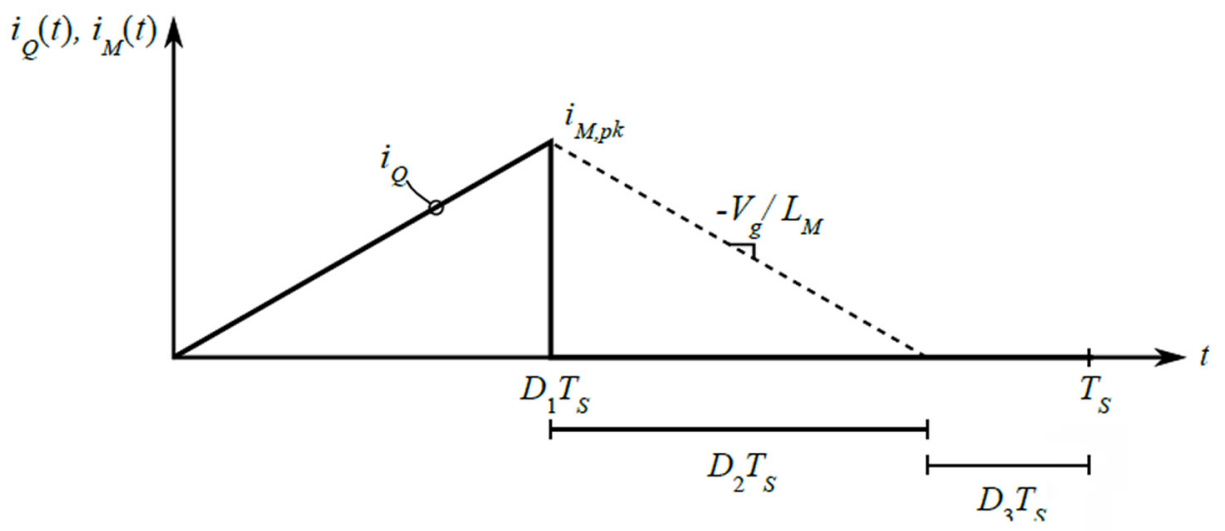

Figura 11. Gráfica para determinar $i_{Q}$. Fuente: Elaboración propia.

De acuerdo a la gráfica de la Figura 11, la corriente $\left\langle i_{Q}\right\rangle$ se determina por:

$$
\begin{aligned}
\left\langle i_{Q}\right\rangle & =\frac{1}{T_{s}} \int_{0}^{T_{s}} i_{Q}(\tau) d t \\
& =\frac{1}{2} \frac{1}{T_{s}} i_{M, p k} D_{1} T_{s} \\
& =\frac{1}{2} i_{M, p k} D_{1}
\end{aligned}
$$

como $i_{M, p k}=D_{2} T_{s}\left(V_{g} / L_{M}\right)$, entonces: 


$$
\begin{aligned}
& \left\langle i_{Q}\right\rangle=\frac{1}{2} D_{1}\left(V_{g} / L_{M}\right) D_{2} T_{S} \\
& \left\langle i_{Q}\right\rangle=\frac{T_{S}}{2 L_{M}} D_{1} D_{2} V_{g}
\end{aligned}
$$

como $\left\langle i_{Q}\right\rangle=\mathrm{V} / \mathrm{R}$, se sustituye en (29):

$$
\frac{V}{R}=\frac{T_{S}}{2 L_{M}} D_{1} D_{2} V_{g}
$$

al despejar $V$ y sustituir la expresión para $D_{2}$, ecuación (21):

$$
\begin{aligned}
& V=\frac{R T_{s}}{2 L_{M}} D_{1} \underbrace{\left(\frac{D_{1}}{V_{g}}\left(V_{g}-V\right)\right)}_{\text {del balancede Volts } / \mathrm{s}} V_{g} \\
& V=\frac{D_{1}^{2}}{K}\left(V_{g}-V\right), \quad \text { con } K=\frac{R T_{s}}{2 L_{M}}
\end{aligned}
$$

Al re-escribir la ecuación (32) se obtiene la expresión (36) para la razón de voltaje del convertidor operando en modo de conducción discontinuo M:

$$
\begin{aligned}
& V=\frac{D_{1}^{2}}{K}\left(V_{g}-V\right) \\
& \frac{K}{D_{1}^{2}} V+V=V_{g} \\
& \left(1+\frac{K}{D_{1}^{2}}\right) V=V_{g} \\
& M\left(D_{1}\right)=\frac{V}{V_{g}}=\frac{1}{1+K / D_{1}^{2}}
\end{aligned}
$$

En la Figura 12 se grafica la relación $M\left(D_{1}\right)$ para diferentes valores de $K$. Note que conforme $K$ es menor, el intervalo de valores de $D_{1}$ en el cual el convertidor está en modo de conducción discontinuo es mayor. Los círculos negros sólidos representan el punto donde el convertidor deja de operar en MCD y por lo tanto se debe utilizar la expresión analítica de modo de conducción continuo (MCC). 


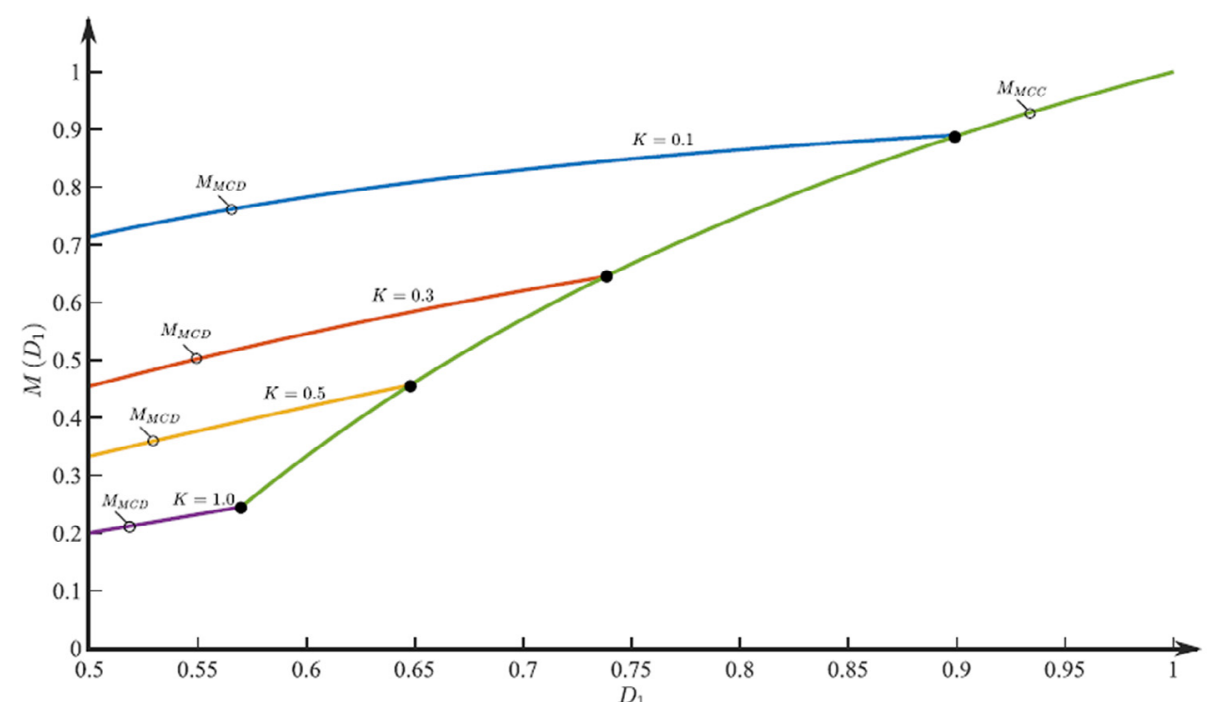

Figura 12. Razón de Conversión de Voltajes $M\left(D_{1}\right)$ del convertidor en MCD. Fuente: Elaboración propia.

\section{EJEMPLO NUMÉRICO Y SIMULACIÓN}

Se muestra un ejemplo numérico y la simulación para verificar la relación de entrada y salida obtenida, así como la corriente de magnetización. Si se tienen los siguientes valores: $V_{g}=48$ Volts $\mathrm{DC}, f_{s}=15 \mathrm{kHz}, D=3 / 4, L_{M}=10 \mathrm{u} H, \mathrm{R}=4 \Omega$ y C $=470 \mathrm{u} F$, el cálculo de la tensión DC V y la corriente $i_{M, p k}$ se obtiene de la siguiente manera:

$$
\begin{aligned}
& K=\frac{2 L_{M}}{R T_{S}}=\frac{2\left(10 \times 10^{-6} \mathrm{H}\right)}{4(15000 \mathrm{~Hz})^{-1}}=0.075 \\
& K_{\text {crit }}=\frac{D^{\prime} D^{2}}{2 D-1}=\frac{(1 / 4)(3 / 4)^{2}}{2(3 / 4)-1}=0.281 \\
& V=\frac{V_{g}}{1+K / D_{1}^{2}}=\frac{48}{1+(0.075) /(3 / 4)^{2}}=42.353 \\
& D_{2}=\frac{D_{1}}{V_{g}}\left(V_{g}-V\right)=\frac{(3 / 4)}{48}(48-42.353)=0.088 \\
& i_{M, p k}=D_{2} T_{S}\left(V_{g} / L_{M}\right)=(0.088)(15000)^{-1}\left(48 /\left(10 \times 10^{-6}\right)\right)=28.240
\end{aligned}
$$

Dado que $K<K_{\text {crit }}$, entonces la inecuación (10) es verdadera y por lo tanto se está operado en Modo de Conducción Discontinuo. En la Figura 13 se muestra el circuito simulado en el ambiente Gecko Circuits. La simulación se muestra en la Figura 14. Los valores simulados obtenidos son: $V=42.464, i_{M, p k}=27.987, D_{2}=0.087$. 


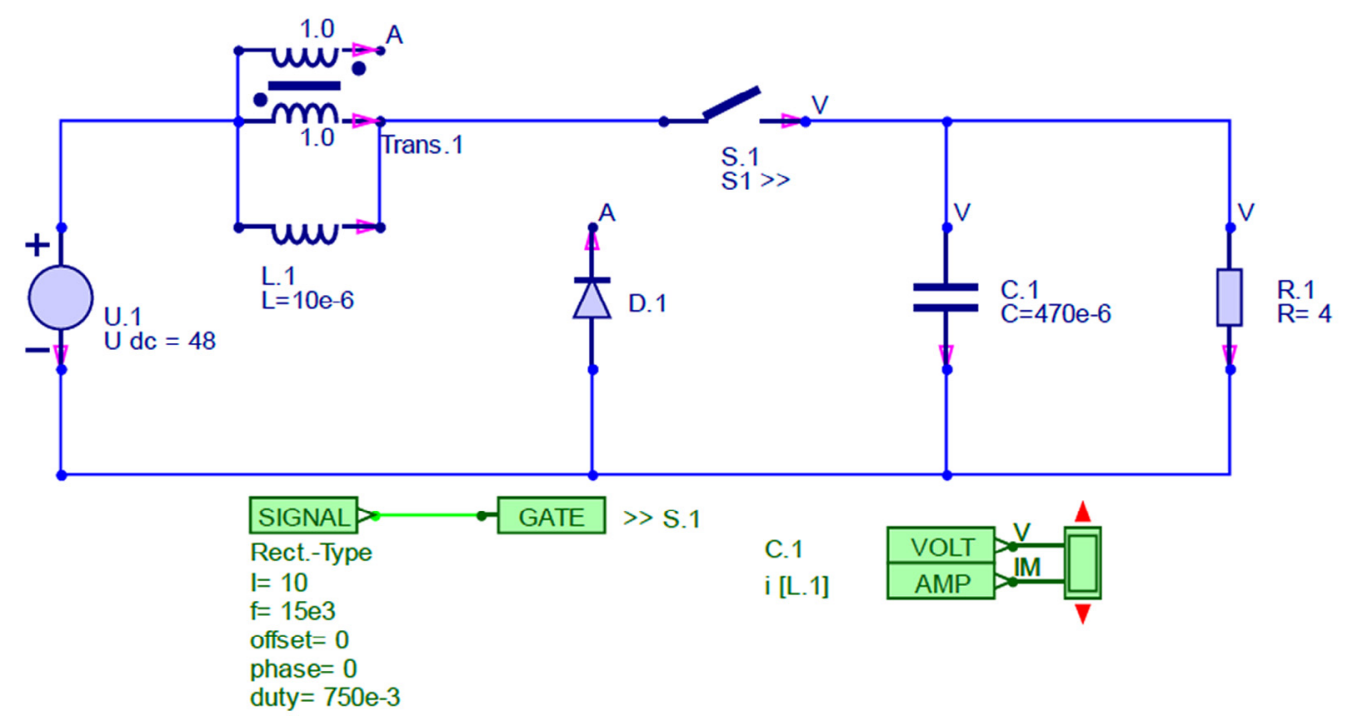

Figura 13. Circuito simulado en Gecko Circuits. Fuente: Elaboración propia.

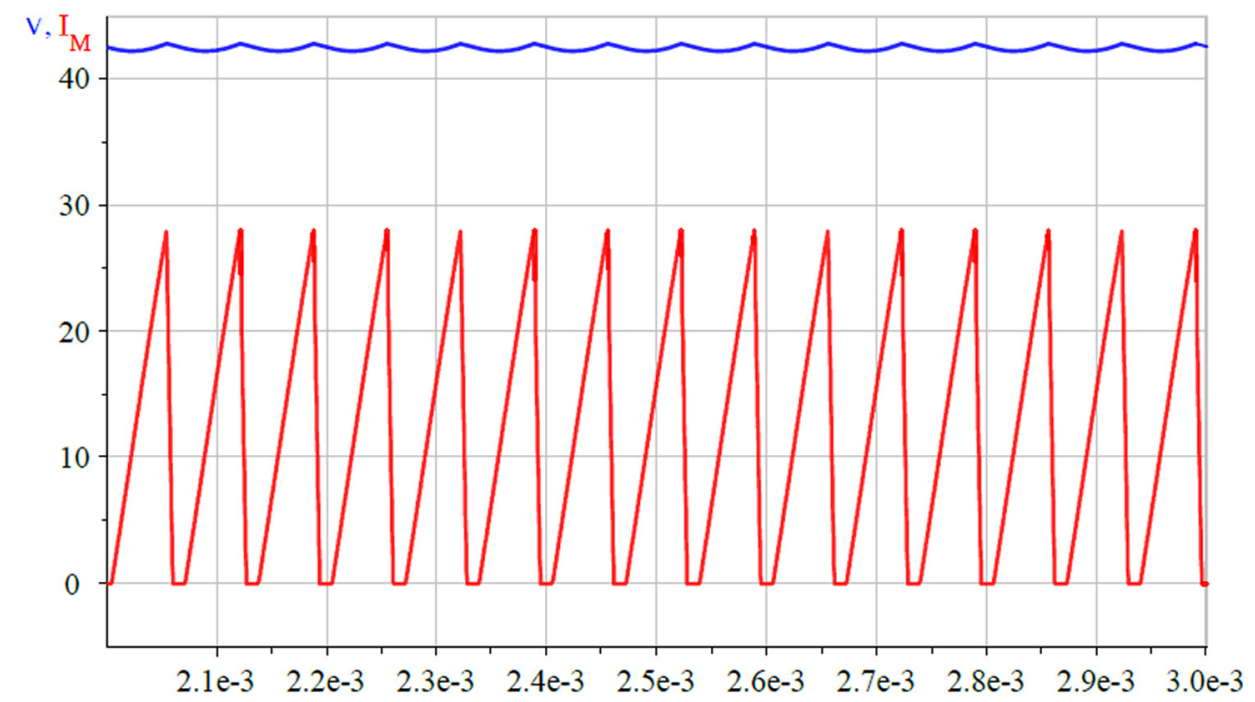

Figura 14. Ondas de tensión de salida y de corriente de magnetización simuladas en Gecko Circuits. Fuente: Elaboración propia.

\section{CONCLUSIONES}

El análisis del modo de conducción discontinuo en el convertidor Flyback ${ }^{-1}$ con $n=1$ muestra que tiene un comportamiento tipo buck al igual que en modo de conducción continuo, pero con una menor pendiente de crecimiento por incremento de ciclo de trabajo. El comportamiento se reconoce como tipo buck ya que disminuye el voltaje de salida con respecto al voltaje de entrada.

Dado que la corriente del inductor es pulsante, se recomienda implementar el convertidor con inductores de bajas pérdidas en el cobre, ya que la eficiencia será proporcional a ésta. De 
esta forma, la eficiencia global mejora considerablemente, ya que las pérdidas en los dispositivos seminconductores se minimizan inherentemente al conmutador con cero corriente.

La simulación corroboró exitosamente el análisis, permitiendo establecer adicionalmente la fundación para análisis posteriores con relaciones de vuelta distintos de uno en el transformador. Dependiento de la aplicación, se valora el convertidor Flyback ${ }^{-1}$ para determinar la eficiencia ante otras soluciones, incluyendo el convertidor mismo con diferentes números de vuelta.

Para que el convertidor opere siempre en modo de conducción discontinuo se recomienda diseñar a máxima carga con el valor de la resistencia más bajo. Así, se mantendrá en modo de conducción discontinuo a cargas más bajas (con valores de resistencia de carga más altos). También se puede implementar estrategias de control que modifiquen la frecuencia de conmutación para mantener la operación en modo de conducción discontinuo ante variaciones de la carga.

\section{REFERENCIAS}

Erickson, R. \& Maksimovic, D. (2001). Fundamentals of power electronics. Norwell, MA: Kluwer Academic Publishers.

Gólcher, L (2014). Nuevo Convertidor DC/DC 1-FB-1 . Ingeniería Revista de la Universidad de Costa Rica, $22,13-23$.

Krein, P. (1998). Elements of Power Electronics (First Edition). Oxford: Oxford University Press, Inc.

Middlebrook, R. \& Çuk, S. (1983). Advances in Switched-Mode Power Conversion. Pasadena, CA: TESLAco.

Lin, F. \& Chen, D. (1994). Reduction of power supply EMI emission by switching frequency modulation. IEEE Transanctions Power Electronics., 9, 132-137.

Mohan, N., Undeland, T. \& Robbins, J. (2003). Power Electronics Converters, Applications and Design. Danvers, MA: John Wiley \& Sons, Inc.

Motto, J. (1977). Introduction to Solid State Power Electronics (First Edition). Virginia: Powerex Semiconductor Division.

Nandankar, P. y Rothe, J. (2016). Highly efficient discontinuous mode interleaved dc-dc converter. International Conference on Electrical, Electronics, and Optimization Techniques, 978-1-4673-9939-5/16.

Rashid, M. (1995). Power Electronics Circuits, Devices and Applications (Second Edition). New Jersey: Prentice Hall.

Tse, K., Shu-hung, H., Hui, S. \& So, H. (2000). Spectral Characteristics of Randomly Switched PWM DC/ DC Converters Operating in Discontinuous Conduction Mode. IEEE TRANSACTIONS ON INDUSTRIAL ELECTRONICS, 47(4), 759-769. 